\title{
RESEARCH ARTICLE SUMMARY
}

VACCINOLOGY

\section{De novo protein design enables the precise induction of RSV-neutralizing antibodies}

Fabian Sesterhenn*, Che Yang*, Jaume Bonet $\dagger$, Johannes T. Cramer $\dagger$, Xiaolin Wen, Yimeng Wang, Chi-I Chiang, Luciano A. Abriata, Iga Kucharska, Giacomo Castoro, Sabrina S. Vollersł, Marie Galloux, Elie Dheilly, Stéphane Rosset, Patricia Corthésy, Sandrine Georgeon, Mélanie Villard,

Charles-Adrien Richard, Delphyne Descamps, Teresa Delgado, Elisa Oricchio,

Marie-Anne Rameix-Welti, Vicente Más, Sean Ervin, Jean-François Eléouët, Sabine Riffault, John T. Bates, Jean-Philippe Julien, Yuxing Li, Theodore Jardetzky, Thomas Krey, Bruno E. Correia $\S$

INTRODUCTION: The ultimate goal of de novo protein design is to create proteins endowed with new biological functions. From a structural perspective, this remains a challenge because most biological functions in natural proteins are mediated by irregular and discontinuous structural motifs. By contrast, state-of-the art techniques for de novo protein design excel at designing highly regular structures. Thus, most de novo proteins designed so far are either functionless or present functions that are encoded by regular, continuous secondary structures.

A promising application for de novo proteins is in vaccine design, more specifically the design of proteins that mimic a viral epitope outside the context of the native protein. These proteins, when used as immunogens, have shown promise in inducing targeted virusneutralizing antibodies (nAbs) in vivo. To date, epitope-focused immunogens have been limited to single epitopes that are regular and continuous, greatly limiting their potential in the field of vaccine design.

RATIONALE: A major bottleneck for the design of proteins endowed with complex functional motifs is the lack of appropriate design templates in the known structural repertoire. Here, we propose a strategy to assemble protein topologies tailored to the functional motif with

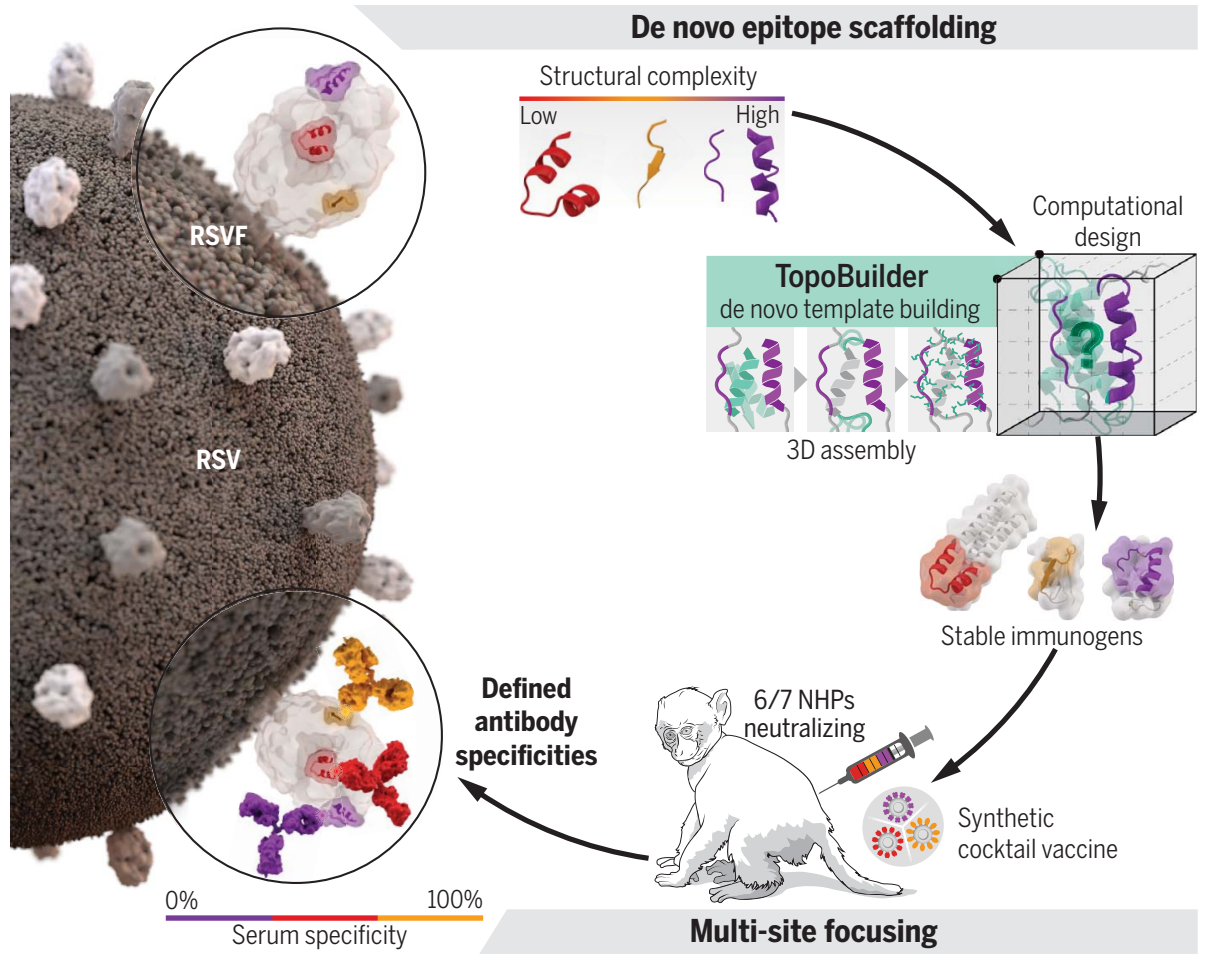

De novo design of a trivalent cocktail vaccine. Structurally complex RSV neutralization epitopes were stabilized in de novo-designed proteins. The computational tool TopoBuilder builds customized protein topologies to stabilize functional structural motifs, followed by folding and sequence design using Rosetta. In vivo, a three-scaffold cocktail induced focused RSV nAbs against the target epitopes in mice and NHPs. the ultimate aim of enabling the design of de novo proteins endowed with complex structural motifs. We sought to apply this approach to develop an immunogen cocktail presenting three major antigenic sites of the respiratory syncytial virus (RSV) fusion protein (RSVF), aiming to induce nAbs acting through precisely defined epitopes.

RESULTS: We developed a novel computational design strategy, TopoBuilder, to build de novo proteins presenting complex structural motifs. TopoBuilder enabled us to define and build protein topologies to stabilize functional motifs, followed by in silico folding and sequence design using Rosetta.

In vitro, the computationally designed proteins bound with high affinity to a panel of human, site-specific RSV nAbs. High-resolution crystal structures of the designs confirmed the atomic-level accuracy of the models and the presented neutralization epitopes.

In vivo, cocktail formu-

\section{ON OUR WEBSITE}

Read the full article at https://dx.doi. org/10.1126/ science.aay5051 lations of the immunogens ("Trivax") induced a balanced antibody response targeting three defined epitopes, yielding neutralizing serum levels in mice and nonhuman primates

(NHPs) after a single boost. Trivax elicited a remarkably focused immune response toward the target antigenic sites. Moreover, when used as a boosting immunogen after prefusion RSVF administration, Trivax profoundly reshaped the serum composition, leading to a higher fraction of epitope-specific antibodies and an increased quality of the antibody response compared with prefusion RSVF boosting immunizations. At the molecular level, monoclonal antibodies isolated from Trivax-immunized NHPs were epitope specific, and in one instance resembled those induced by viral infection in humans.

CONCLUSION: Our work provides a new route to functionalizing de novo proteins and presents a blueprint for epitope-centric vaccine design, offering an unprecedented level of control over induced antibody specificities in both naïve and primed antibody repertoires. Beyond immunogens, the ability to design de novo proteins presenting functional sites with high structural complexity will be broadly applicable to expanding the structural and sequence repertoires, but above all, the functional landscape of natural proteins.

The list of author affiliations is available in the full article online. *These authors contributed equally to this work †These authors contributed equally to this work. †Present address: Ichnos Sciences SA, La Chaux-de-Fonds Switzerland.

§Corresponding author. Email: bruno.correia@epfl.ch Cite this article as F. Sesterhenn et al., Science 368, eaay5051 (2020). DOI: 10.1126/science.aay5051 


\section{RESEARCH ARTICLE}

VACCINOLOGY

\section{De novo protein design enables the precise induction of RSV-neutralizing antibodies}

\author{
Fabian Sesterhenn ${ }^{1,2 *}$, Che Yang ${ }^{1,2 *}$, Jaume Bonet ${ }^{1,2} \dagger$, Johannes T. Cramer ${ }^{3}$, Xiaolin Wen ${ }^{4}$, \\ Yimeng Wang ${ }^{5}$, Chi-I Chiang ${ }^{5}$, Luciano A. Abriata ${ }^{1,2}$, Iga Kucharska ${ }^{6,7}$, Giacomo Castoro ${ }^{3}$, \\ Sabrina S. Vollers ${ }^{1,2} \neq$, Marie Galloux ${ }^{8}$, Elie Dheilly ${ }^{9}$, Stéphane Rosset ${ }^{1,2}$, Patricia Corthésy ${ }^{1,2}$, \\ Sandrine Georgeon ${ }^{1,2}$, Mélanie Villard ${ }^{1,2}$, Charles-Adrien Richard ${ }^{8}$, Delphyne Descamps ${ }^{8}$, \\ Teresa Delgado ${ }^{10}$, Elisa Oricchio ${ }^{9}$, Marie-Anne Rameix-Welti ${ }^{11}$, Vicente Más ${ }^{10}$, Sean Ervin $^{12}$, \\ Jean-François Eléouët ${ }^{8}$, Sabine Riffault ${ }^{8}$, John T. Bates ${ }^{13}$, Jean-Philippe Julien ${ }^{6,7}$, Yuxing Li ${ }^{5,14}$, \\ Theodore Jardetzky ${ }^{4}$, Thomas Krey, $3,15,16,17,18$, Bruno E. Correia ${ }^{1,2} \S$
}

De novo protein design has been successful in expanding the natural protein repertoire. However, most de novo proteins lack biological function, presenting a major methodological challenge. In vaccinology, the induction of precise antibody responses remains a cornerstone for next-generation vaccines. Here, we present a protein design algorithm called TopoBuilder, with which we engineered epitope-focused immunogens displaying complex structural motifs. In both mice and nonhuman primates, cocktails of three de novo-designed immunogens induced robust neutralizing responses against the respiratory syncytial virus. Furthermore, the immunogens refocused preexisting antibody responses toward defined neutralization epitopes. Overall, our design approach opens the possibility of targeting specific epitopes for the development of vaccines and therapeutic antibodies and, more generally, will be applicable to the design of de novo proteins displaying complex functional motifs.

I he computational design of new proteins from first principles has revealed a variety of rules for the accurate design of structural features in de novo proteins (1-4). However, the de novo design of functional proteins remains far more challenging $(5,6)$. A commonly used strategy to design functional proteins is to transplant structural motifs from other proteins into preexisting or de novo protein scaffolds (7-9). In nearly all cases previously reported, the transplanted motifs mediated protein-protein interactions. These structural motifs are common in the natural protein repertoire, for example, in linear helical segments, which allows their grafting without extensive backbone adjustments $(7,8)$. Most protein functional sites, however, are not contained within regular single segments in protein structures but rather arise from the three-dimensional (3D) arrangement of multiple, and often irregular, structural elements supported by the overall architecture of the protein structure (10-12). Therefore, the development of computational approaches to endow de novo proteins with irregular and multisegment motifs is crucial to expanding their function and the scope of their application.

Protein design has sparked hopes in the field of rational vaccinology, particularly to elicit targeted neutralizing antibody $(\mathrm{nAb})$ responses $(9,13)$. Although many potent nAbs have been identified and structurally characterized in complex with their target antigens, the design of immunogens that elicit precise and focused antibody responses remains a major challenge $(14,15)$. To date, structurebased immunogen design efforts have mostly focused on modifying viral fusion proteins through conformational stabilization, silencing of non-neutralizing epitopes, and targeting the germline precursors of nAbs. (16). Unlike respiratory syncytial virus (RSV), several major human pathogens only display a limited number of broadly neutralizing epitopes that are surrounded by strain-specific, non-neutralizing, or disease-enhancing epitopes (17-19). Thus, one of the central goals for vaccine development is to elicit antibody responses with precisely defined epitope specificities and, in some cases, constrained molecular features (e.g., antibody lineage, complementarity-determining region length, or binding angle) (20-24).

The difficulty in developing immunogens that can elicit antibodies specific for a restricted subset of epitopes on a single protein continues to be a critical barrier to rational vaccine design. Previous studies have sought to elicit epitope-specific responses using peptidebased approaches (25) or epitope scaffolds $(9,13,26-28)$. Leveraging computational design, the antigenic site II of the RSV fusion protein (RSVF), a linear helix-turn-helix motif, was transplanted onto a heterologous protein scaffold, which was shown to elicit nAbs in nonhuman primates (NHPs) after repeated boosting immunizations (9). Despite this proofof-principle showing the induction of functional antibodies using a computationally designed immunogen, two major caveats have emerged: the neutralization titers observed in immunogenicity studies were inconsistent and the computational approach was not suitable for structurally complex epitopes.

To address these limitations, we used de novo design approaches to engineer epitope-focused immunogens mimicking irregular and discontinuous RSV neutralization epitopes [sites 0 (29) and IV (30); Fig. 1]. We designed a trivalent cocktail formulation ("Trivax") consisting of a previously published immunogen for site II (13) and in-house-designed immunogens mimicking sites 0 and IV. In vivo, Trivax induced a balanced antibody response against all three epitopes, resulting in consistent levels of serum neutralization in six of seven NHPs. Upon priming with RSVF, the computationally designed immunogens boosted site-specific antibodies, resulting in an improved antibody quality. Our approach enables the targeting of specific epitopes for the development of vaccines and therapeutic antibodies and, more broadly, will be applicable in the design of de novo proteins displaying complex functional motifs.

\section{Results}

\section{De novo design of immunogens presenting} structurally complex epitopes

The computational design of proteins mimicking structural motifs has been done previously by first identifying compatible protein scaffolds,

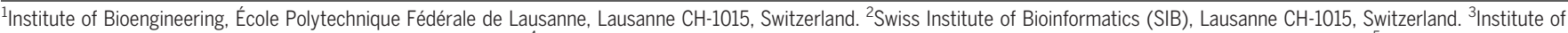

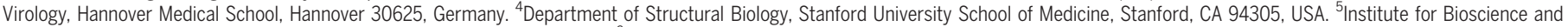

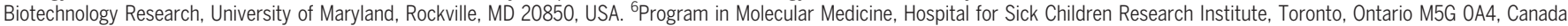

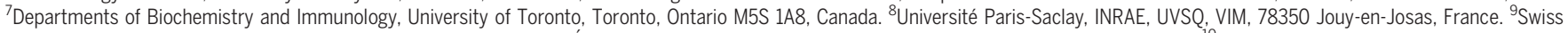

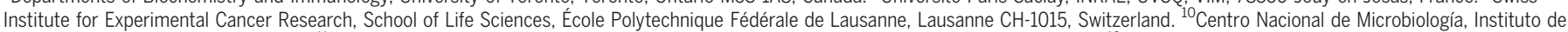

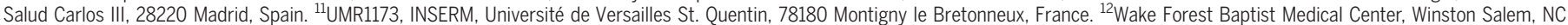

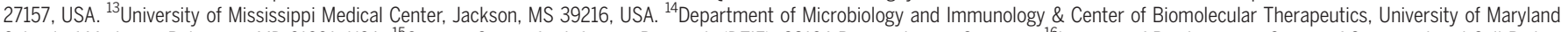

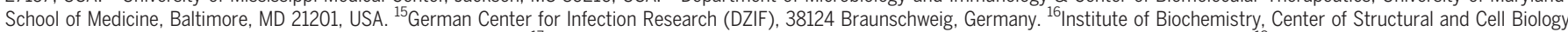

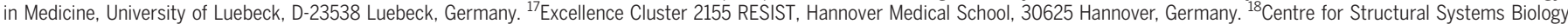
(CSSB), 22607 Hamburg, Germany.

*These authors contributed equally to this work. †These authors contributed equally to this work. łPresent address: Ichnos Sciences SA, La Chaux-de-Fonds, Switzerland. §Corresponding author. Email: bruno.correia@epfl.ch
} 
A

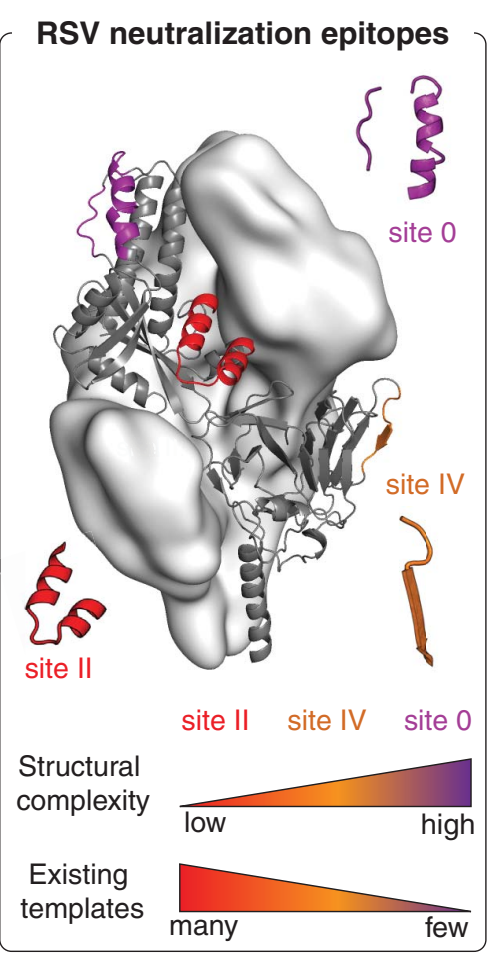

B

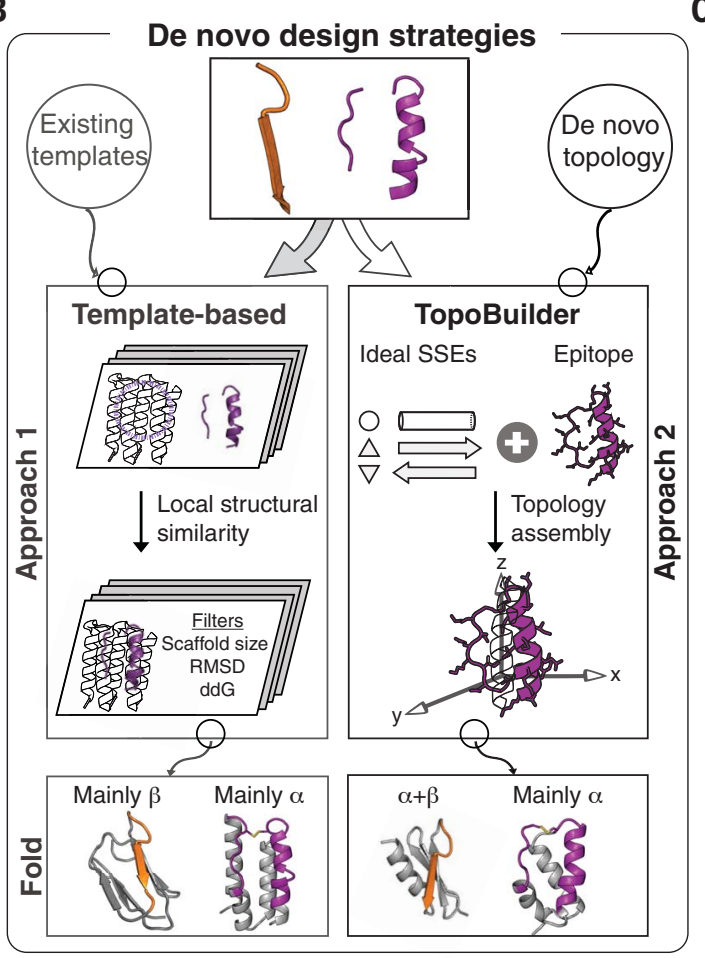

C

Functional evaluation in vivo

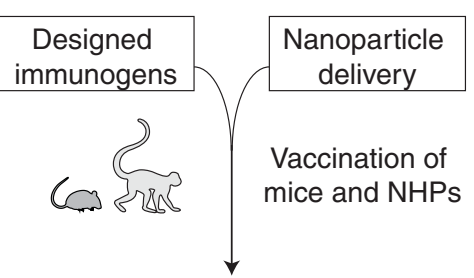

Multi-site immunofocusing

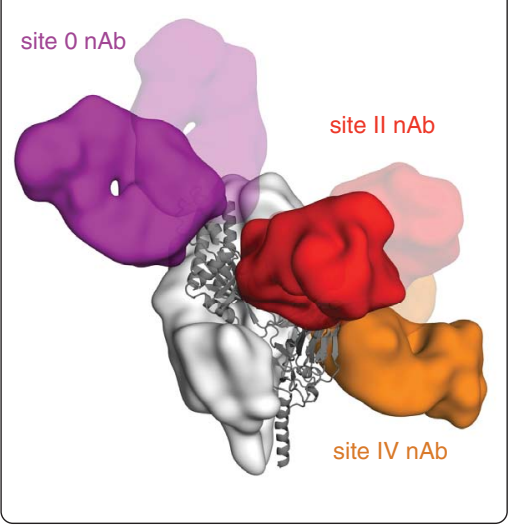

Fig. 1. Computational design of RSV epitope-focused immunogens. (A) Prefusion RSVF structure (PDB 4JHW) with sites 0, II, and IV highlighted. (B) Computational protein design strategies. Approach 1: Design templates were identified in the PDB, followed by in silico folding and design. Approach 2: A template-free design approach ("TopoBuilder") was developed to tailor the protein topology to the motif's structural constraints. At the bottom are computational models of the designed immunogens. $\alpha$, alpha helix; $\beta$, beta strand; ddG, computed binding energy. (C) Cocktail formulations of designed immunogens displayed on nanoparticles elicit nAbs focused on three nonoverlapping epitopes.

which then serve as design templates to graft the motifs $(7,26-28,31,32)$. This approach, referred to as template-based design, has been used to transplant functional sites both to structures from the natural repertoire $(26-28,31,32)$ and to those from de novodesigned proteins (7). Although most studies have focused on linear, regular binding motifs, one study successfully grafted a structurally complex HIV epitope into an existing protein scaffold. However, both the overall structure and sequence of the template remained mostly native (31).

Here, we sought to design accurate mimetics of RSVF neutralization epitopes based on de novo proteins and evaluate their functionality in immunization studies. We chose antigenic sites 0 and IV (Fig. 1A), which are both targeted by potent nAbs and have a high structural complexity; site 0 is a discontinuous epitope consisting of a kinked, 17-residue alpha helix and a disordered, seven-residue loop $(29,33)$, whereas site IV presents an irregular, six-residue, bulged beta strand (30).

In a first effort, we used a template-based de novo design approach relying on Rosetta FunFolDes (34) to fold and design scaffolds for sites IV and 0 . Given the structural complexity of these sites, few structures in the Protein Data Bank (PDB) matched the backbone con- figuration of the epitopes, even using loose structural criteria (fig. S1). Briefly, our best computational design for site IV (S4_1.1), based on a domain excised from prefusion RSVF (preRSVF), bound with weak affinity to the target $\mathrm{nAb} 101 \mathrm{~F}$ [dissociation constant $\left.\left(K_{\mathrm{D}}\right)>85 \mu \mathrm{M}\right]$. After in vitro evolution, we obtained a double mutant (S4_1.5) that bound $101 \mathrm{~F}$ with a $K_{\mathrm{D}}$ of $35 \mathrm{nM}$ and was thermostable up to $65^{\circ} \mathrm{C}$ (Fig. 2, B to D, and figs. S2 and S3). For site 0 , we used a designed helical repeat protein [PDB 5CWJ (35)] as a design template. Our first computational design showed a $K_{\mathrm{D}}$ of $1.4 \mu \mathrm{M}$ to the target $\mathrm{D} 25 \mathrm{nAb}$, which we improved to a $K_{\mathrm{D}}$ of $5 \mathrm{nM}$ upon several truncations, iterative rounds of computational design, and in vitro evolution (Fig. 2 and figs. S4 and S5).

This template-based approach led to designs that presented several desired features (e.g., stability and antibody binding). However, important limitations emerged during the design process: (i) extensive in vitro evolution optimization was required, (ii) binding affinities to target nAbs were one to two orders of magnitude lower than those of the viral protein (preRSVF), and (iii) suboptimal template topologies constrained the epitope accessibility (fig. S6).

To address these limitations, we developed a template-free design protocol, TopoBuilder, which generates tailor-made protein topol- ogies to stabilize complex functional motifs. TopoBuilder consists of three stages (Fig. 3A). The first stage is topological sampling in 2D space. To quickly define the fold space compatible with the target structural motif, we used the $\alpha \beta \alpha$-Form topology definition scheme, a string-based descriptor that allows the extensive enumeration of multilayer protein topologies with alternating secondary structure elements and all possible connections between the secondary structural elements $(36,37)$. Putative folds were then selected according to basic topological rules (e.g., lack of crossover loops and chain directionality of the functional motif). This allowed the definition of the fold space for a given design task, thereby overcoming a main hurdle in de novo design approaches. The second stage of TopoBuilder is $3 \mathrm{D}$ projection and parametric sampling. The selected 2D topologies were projected into the 3D space by assembling idealized secondary structure elements (SSEs) around the fixed functional motif. These 3D structures, referred to as "sketches," were further refined by coarsely sampling structural features of the fold (e.g., distances and orientations between SSEs) using parametric sampling. The final stage is flexible backbone sampling and sequence design. To refine the structural features of the sketches at the all-atom level and to design sequences that 
A
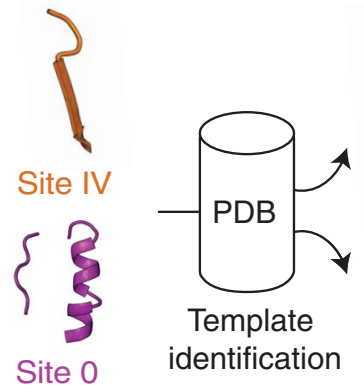

Template identification

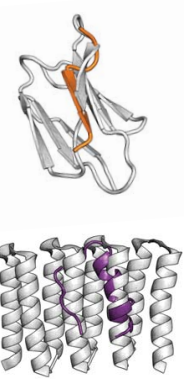

$\stackrel{\mathrm{N}_{\text {mut. }}=40}{\longrightarrow}$

In silico

folding \& design

$\overrightarrow{\mathrm{N}_{\text {trun. }}=31}$

$\mathrm{N}_{\text {mut }}=80$
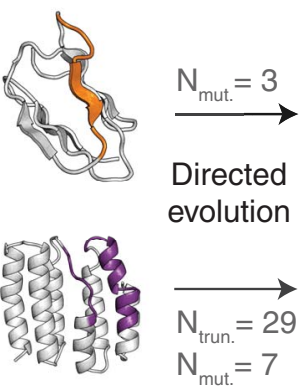

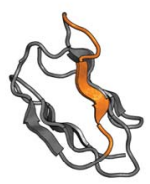

S4_1.05

In silico

folding \& design

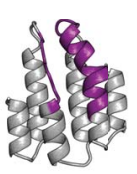

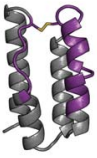

S0_1.39
B

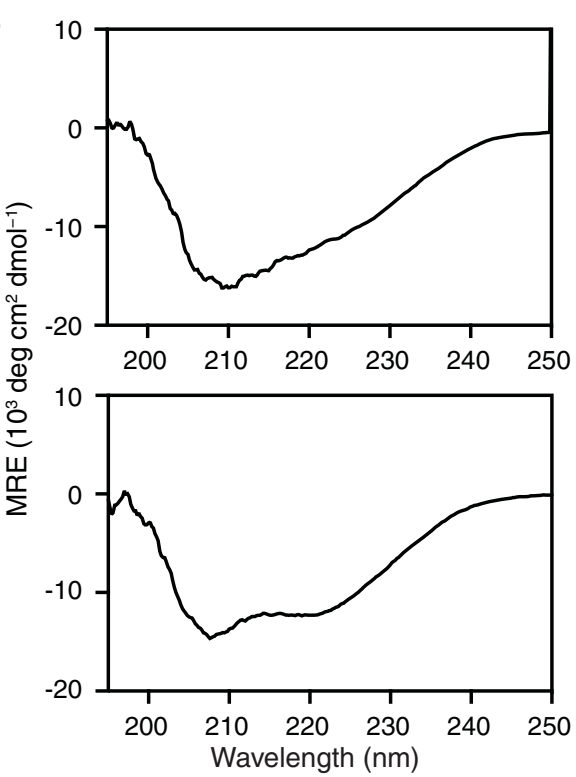

C

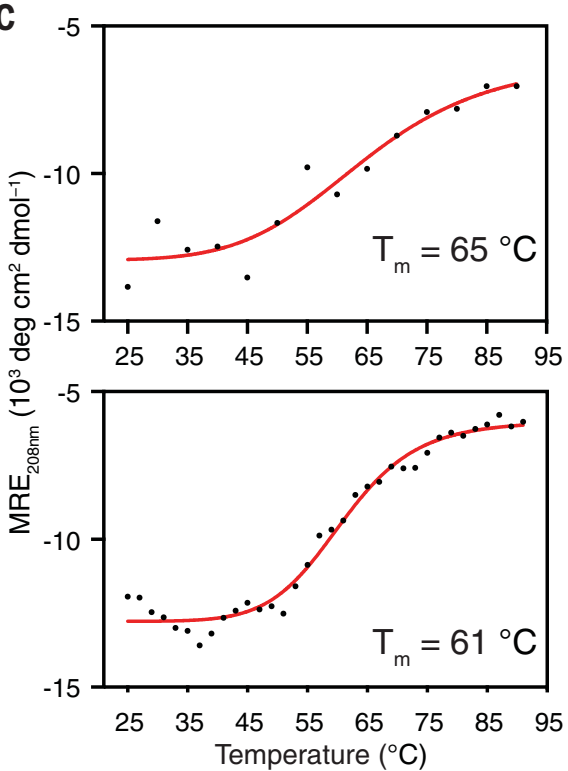

D
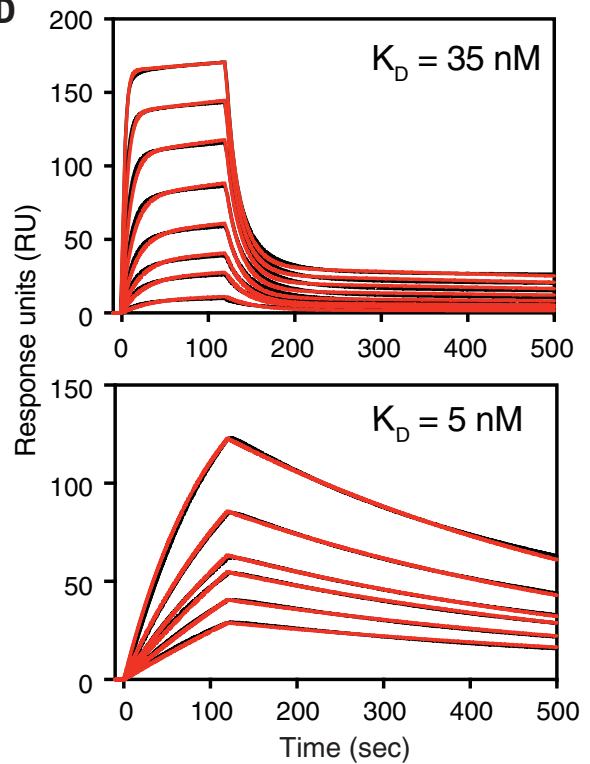

Fig. 2. Template-based computational design. (A) Templates with structural similarity to sites IV and 0 were identified by native domain excision or loose structural matching, followed by in silico folding, design, and directed evolution. Computational models of intermediates and final designs (S4_1.5 and S0_1.39) are shown and the number of mutations ( $\mathrm{N}_{\text {mut. }}$ ) and truncated residues $\left(\mathrm{N}_{\text {trun. }}\right.$ ) are indicated for each step. (B) CD spectra measured at $20^{\circ} \mathrm{C}$ of S4_1.5 (top) and S0_1.39 (bottom) are in agreement with the expected secondary structure content of the design models. MRE, mean residue ellipticity. (C) Thermal melting curves measured by CD in presence of reducing agent. (D) Binding affinity measured by surface plasmon resonance against target antibodies 101F (top) and D25 (bottom). Sensorgrams are shown in black and fits in red. stabilize these structures, we used Rosetta FunFolDes as described previously $(9,34)$.

To present antigenic site IV, we designed a fold composed of a beta sheet with four antiparallel strands and one helix (Fig. 3A), referred to as the S4_2 fold. Within the S4_2 topology, we generated three structural variants (S4 2_bb1 to S4_2_bb3) by sampling three distinct orientations of the helical element, varying both orientations and lengths to optimize the packing interactions with the beta sheet. Sequences generated from two structural variants (S4_2_bb2 and S4_2_bb3) showed a strong propensity to recover the designed structures in Rosetta $a b$ initio simulations (fig. S7).

To evaluate our design approach, we screened a library of designed sequences using yeast display and applied two selective pressures: binding to the $101 \mathrm{~F}$ antibody and resistance to the nonspecific protease chymotrypsin (Fig. 3B), an effective method to digest partially unfolded proteins $(7,38)$. To reveal structural and sequence determinants of designs that led to stable folds and high-affinity binding to 101F, we performed next-generation sequencing of populations sorted under different conditions, retrieving stability and binding scores for each design. We found that S4_2_bb2-based designs were preferentially enriched over the bb1 and bb3 design series, showing that subtle topological differences in the design template can have a substantial impact on function and stability (Fig. 3C). Thirteen of the 14 bestscoring S4_2 variants, bearing between one and 38 mutations compared with each other, were successfully purified and biochemically characterized (fig. S8). The designs showed mixed alpha-beta circular dichroism (CD) spectra and bound to $101 \mathrm{~F}$ with affinities ranging from 1 to $200 \mathrm{nM}$ (fig. S8). The best variant, S4_2.45, was well folded according to $\mathrm{CD}$ and nuclear magnetic resonance (NMR) spectroscopy and only showed partial unfolding even at $90^{\circ} \mathrm{C}$ (Fig. $3 \mathrm{E}$ and fig. S9). S4_2.45 showed a $K_{\mathrm{D}}$ of $1 \mathrm{nM}$ to the target antibody $101 \mathrm{~F}$ (Fig. 3G), consistent with the preRSVF$101 \mathrm{~F}$ interaction $\left(K_{\mathrm{D}}=4 \mathrm{nM}\right)$.

Similarly, we built a minimal de novo topology to present the tertiary structure of the site 0 epitope. The choice for this topology was motivated by the native environment of site 0 in preRSVF, where it is accessible for antibodies with diverse angles of approach (33) (fig. S6). We explored the topological space within the shape constraints of preRSVF and built three different helical orientations ( $\mathrm{SO}_{-}$ 2_bb1 to S0_2_bb3) that supported the epitope segments. Rosetta ab initio folding predictions showed that only designs based on one topology (SO_2_bb3) presented funnel-shaped energy landscapes (fig. S10). A set of computationally designed sequences based on the S0_2_bb3 template was screened in yeast under the 
A 2D topology generation

3D topology assembly bb1 bb2 bb3

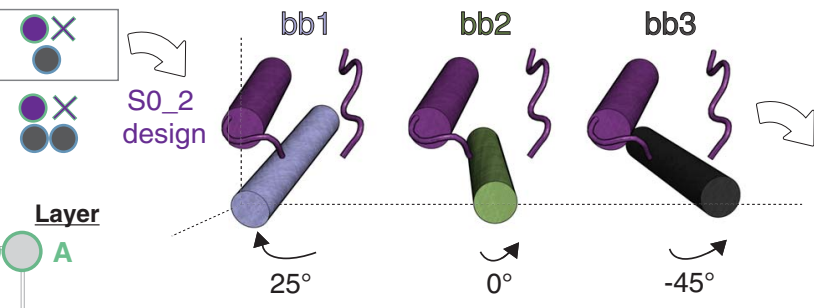

$25^{\circ}$

$\nabla$ в

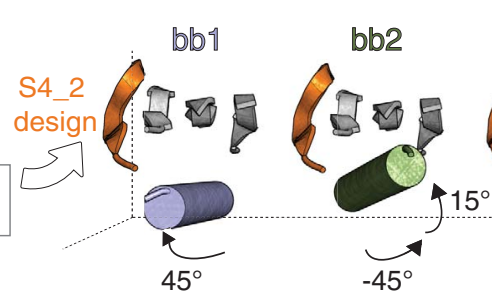

C

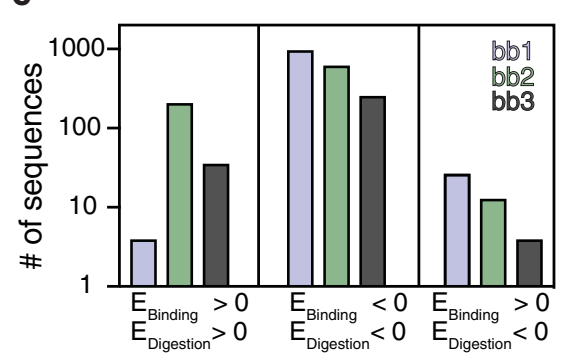

Rosetta FunFolDes

Folding \& sequence design

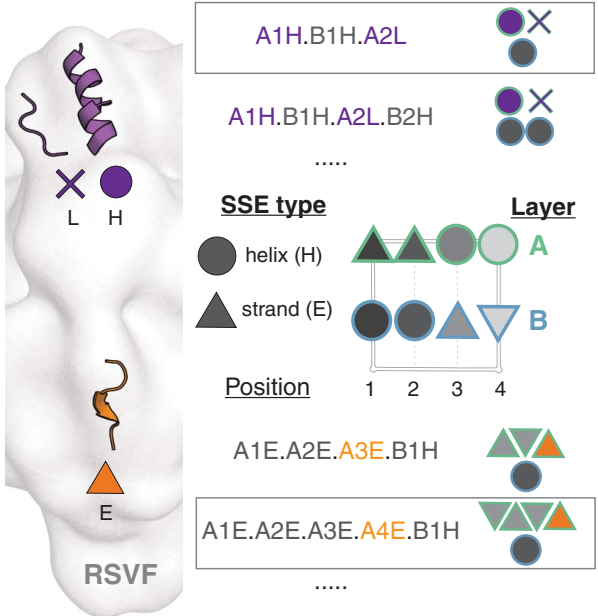

B

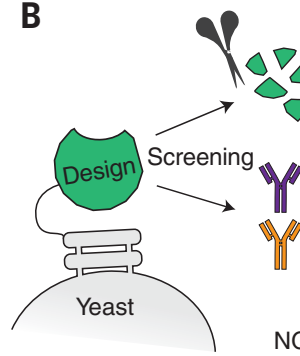

NGS of sorted populations

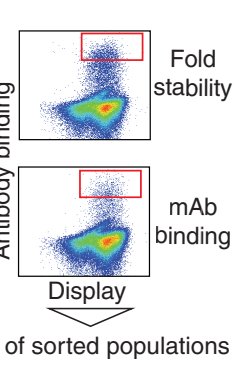

$\mathbf{F}$

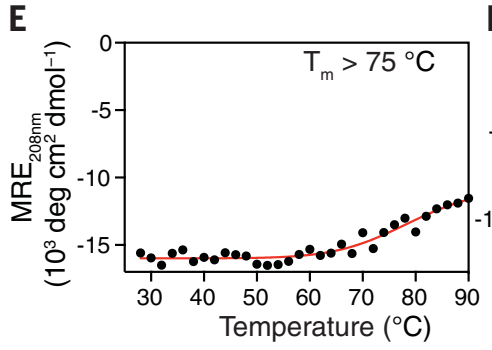

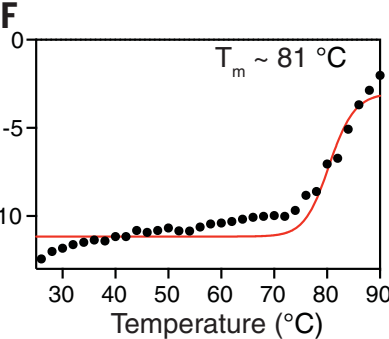

Temperature $\left({ }^{\circ} \mathrm{C}\right)$
D

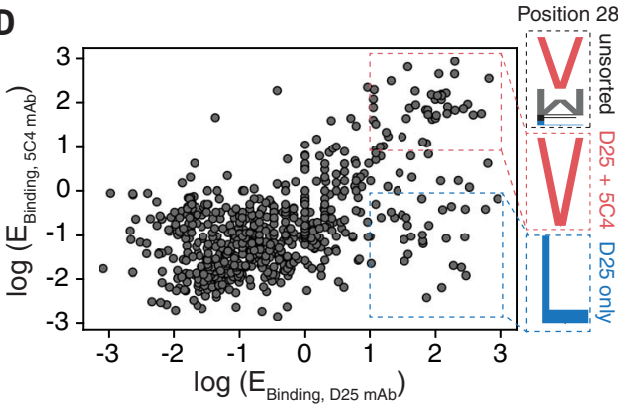

Fig. 3. Template-free de novo design strategy. (A) Protein topologies compatible with each motif are enumerated in the 2D space. Selected topologies are then projected into the 3D space using idealized SSEs and their relative orientation is sampled parametrically. Distance constraints are derived from selected topologies to guide in silico folding and sequence design using Rosetta. (B) Designed sequences were screened for high-affinity binding and resistance to chymotrypsin to select stably folded proteins, as revealed by next-generation sequencing (NGS). (C) For the S4_2 design series, enrichment analysis revealed a strong preference for one of the
G

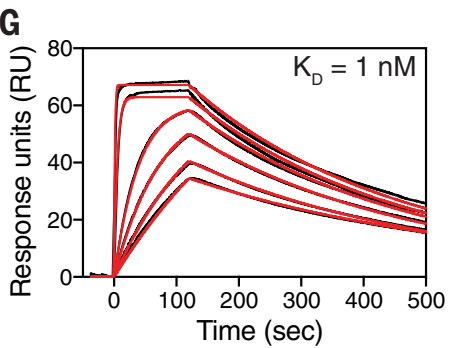

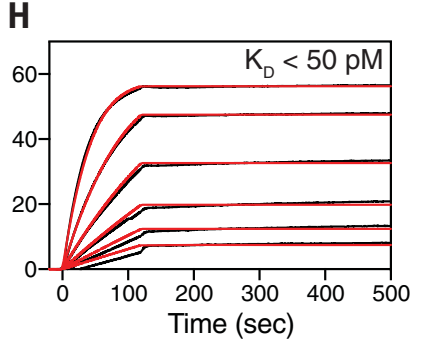

designed helical orientations (S4_2_bb2, green) to resist protease digestion and to bind with high affinity to $101 \mathrm{~F}$. E, enrichment. (D) To ensure epitope integrity, S0_2_bb3 was screened for binding to both D25 and $5 \mathrm{C} 4$. Sequences highly enriched for both D25 and 5C4 binding show convergent sequence features in the critical core position 28 of the site 0 scaffold. (E and $\mathbf{F}$ ) Thermal melting curves measured by $C D$ for best designs [S4_2.45 (E) and S0_2.126 (F)] showing high thermostability. ( $\mathbf{G}$ and $\mathbf{H}$ ) $K_{\mathrm{D}}$ of S4_2.45 to $101 \mathrm{~F}(\mathrm{G})$ and S0_2.126 to D25 (H) as measured by surface plasmon resonance. selective pressure of two site 0 -specific antibodies (D25 and 5C4) to ensure the presentation of the native epitope conformation. Deep sequencing of the double-enriched clones revealed that subtle sequence variants (e.g., position 28) are sufficient to change the antibody-binding properties of the designs, highlighting the challenges of designing functional proteins (Fig. 3D). From the high-throughput screening, we biochemically characterized five sequences bearing between three and 21 mutations compared with each other in a protein of 58 residues (fig. S11). The design with best solution behavior (SO_2.126) showed a CD spectrum of a predominantly helical protein, with extremely high thermostability [melting temperature $\left(T_{\mathrm{m}}\right)=$ $81^{\circ} \mathrm{C}$; Fig. 3F] and a well-dispersed heteronuclear single quantum coherence NMR spectrum (fig. S9). S0_2.126 bound with $K_{\mathrm{D}} \mathrm{S}$ of $\sim 50 \mathrm{pM}$ and $4 \mathrm{nM}$ to $\mathrm{D} 25$ and $5 \mathrm{C} 4$, respective- ly, which is consistent with the affinities of the nAbs to preRSVF ( $150 \mathrm{pM}$ and $13 \mathrm{nM}$ for D25 and $5 \mathrm{C} 4$, respectively) (Fig. $3 \mathrm{H}$ and fig. $\mathrm{S} 12$ ).

Across a panel of site-specific human nAbs (39), S4_2.45 and S0_2.126 showed largeaffinity improvements compared with the first-generation designs, exhibiting a geometric mean affinity closely resembling that of the antibodies to preRSVF (fig. S12). These results suggest that the immunogens designed 


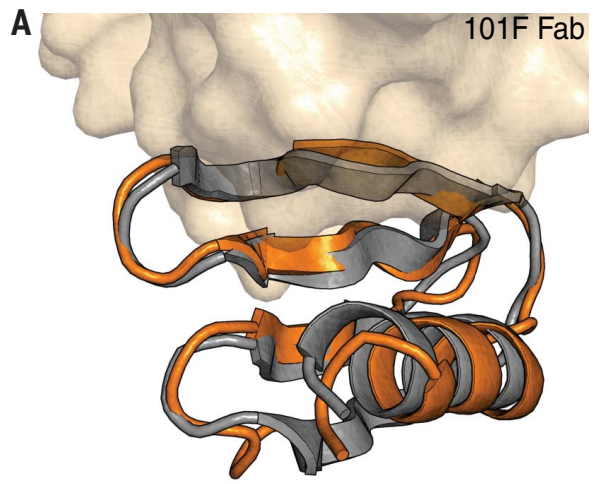

$\begin{array}{ll}\text { Xray structure } & \mathrm{RMSD}=1.5 \AA \\ \text { Computational model } & \mathrm{E} \text { value }=4.8\end{array}$
B

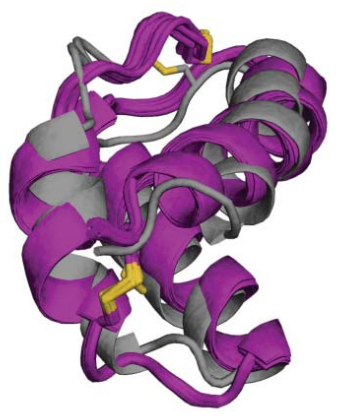

NMR ensemble $\quad$ RMSD $=2.9 \AA$ Computational model
C

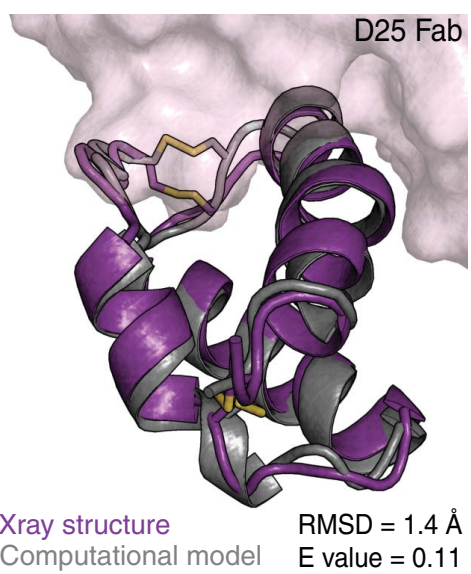

D

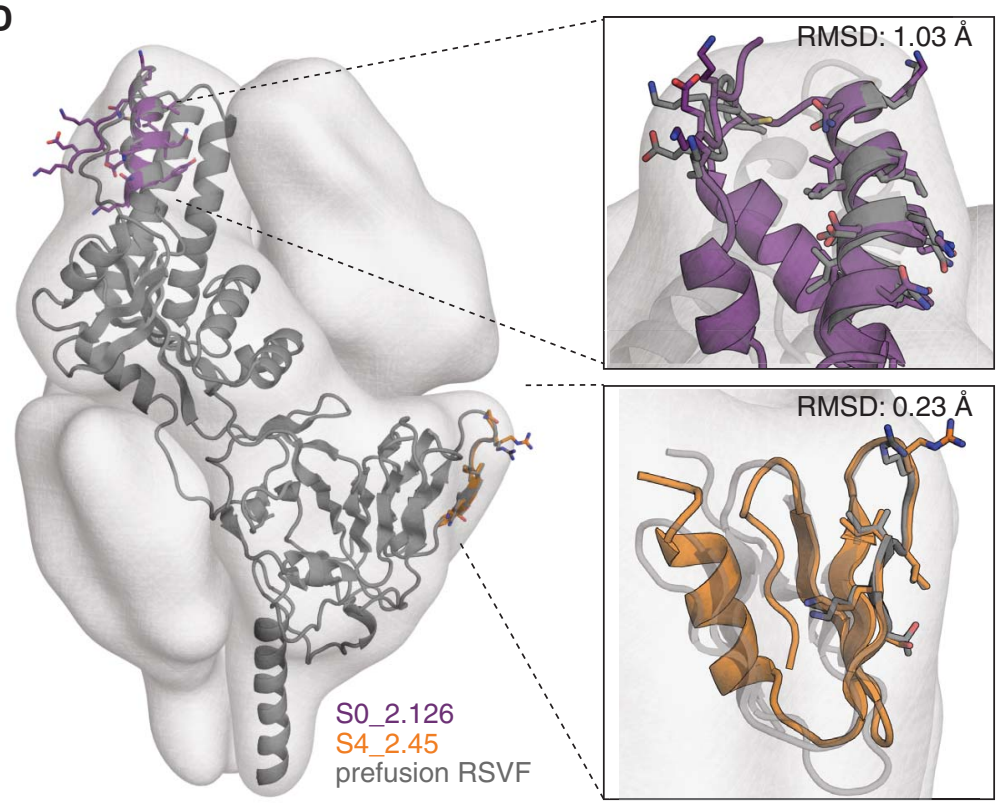

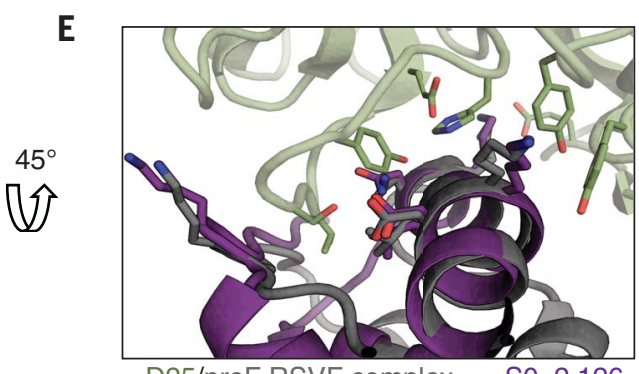

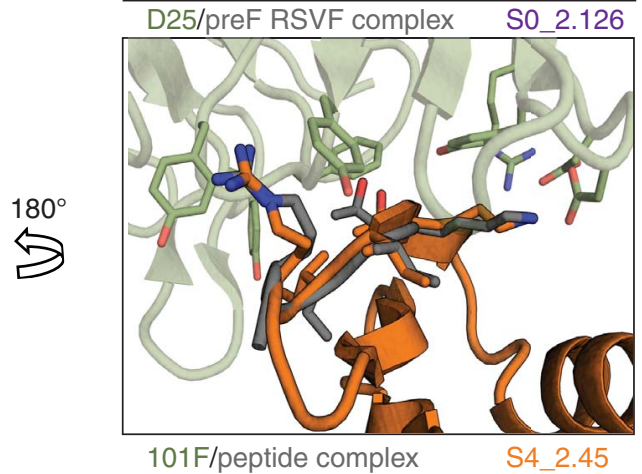

Fig. 4. Structural characterization of de novo-designed immunogens.

(A) Crystal structure of S4_2.45 (orange) bound to 101F Fab closely matches the design model (gray, RMSD = $1.5 \AA$ ). (B) NMR structural ensemble of S0_2.126 (purple) superimposed on the computational model (gray). The NMR structure shows overall agreement with the design model (backbone RMSD of $2.9 \AA$ ). (C) Crystal structure of S0_2.126 (purple) bound to D25 Fab closely resembles the design model (gray, RMSD $=1.4 \AA$ ). (D) Superposition of the preRSVF sites 0 and IV and designed immunogens. Designed scaffolds are compatible with the shape constraints of preRSVF (surface representation). (E) Close-up view of the interfacial side chain interactions between D25 (top) and 101F (bottom) with designed immunogens compared with the starting epitope structures. using TopoBuilder were superior mimetics of sites IV and 0 compared with the templatebased designs.

\section{De novo-designed topologies adopt the predicted structures with high accuracy}

To evaluate the structural accuracy of the computational design approach, we solved the crystal structure of S4_2.45 in complex with $101 \mathrm{~F}$ at $2.6 \AA$ resolution. The structure closely resembled our design model, with a backbone root mean square deviation (RMSD) of $1.5 \AA$ (Fig. 4A). The epitope was mimicked with an RMSD of $0.23 \AA$ and retained all essential interactions with $101 \mathrm{~F}$ (Fig. 4, D and E, and fig. S13). The structural data confirmed that we accurately presented an irregular beta strand, a common motif found in many proteinprotein interactions (40), in a fully de novodesigned protein with sub-ångström accuracy.

Next, we solved an unbound structure of S0_2.126 by NMR, confirming the accuracy of the designed fold with a backbone RMSD of $2.9 \AA$ between the average structure and the computational model (Fig. 4B). Additionally, we solved a crystal structure of S0_2.126 bound to D25 at a resolution of $3.0 \AA$. The structure showed backbone RMSDs of $1.4 \AA$ to the design model and $1.03 \AA$ over the discontinuous epitope compared with preRSVF (Fig. 4, C to E, and fig. S13). Compared with native proteins, S0_2.126 showed exceptionally low core packing because of a large cavity (fig. S14) but retained a very high thermal stability. The core cavity was essential for antibody binding and highlights the potential of de novo approaches to design small proteins hosting structurally challenging motifs and preserving cavities required for function (2). 
A

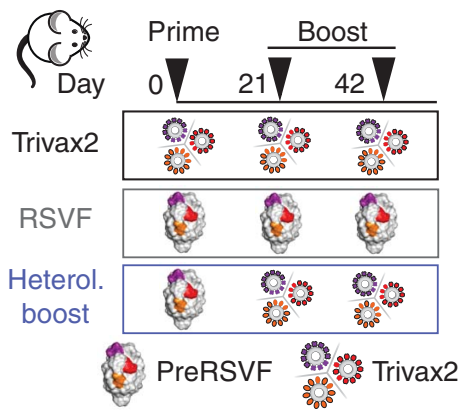

B

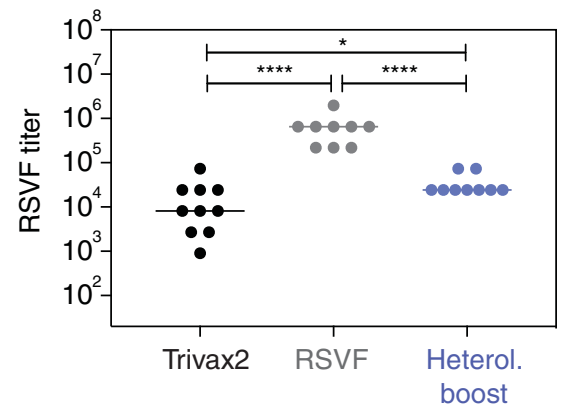

C

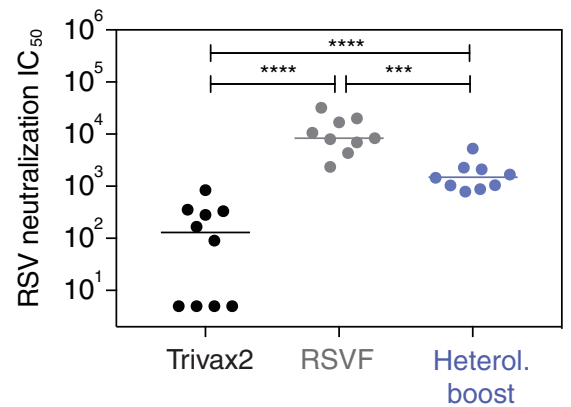

D

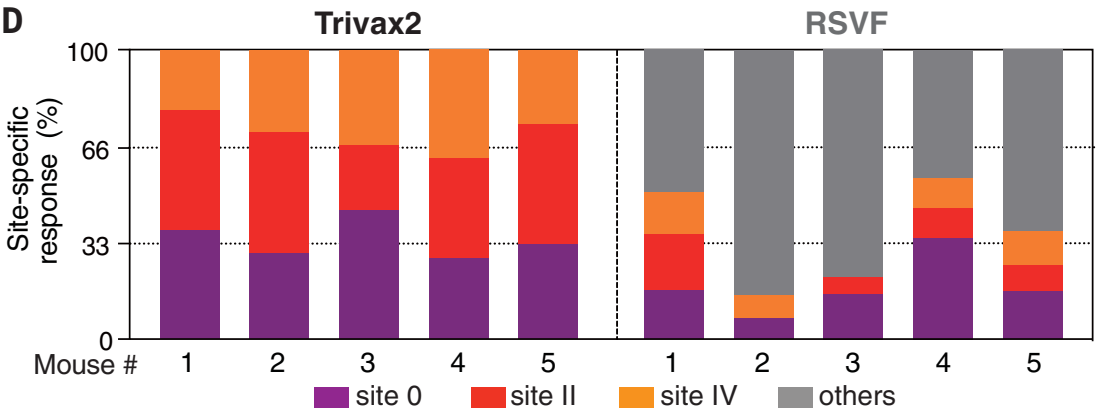

$\mathbf{E}$

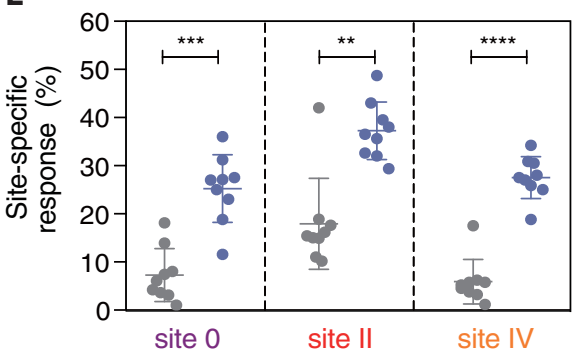

$\mathbf{F}$

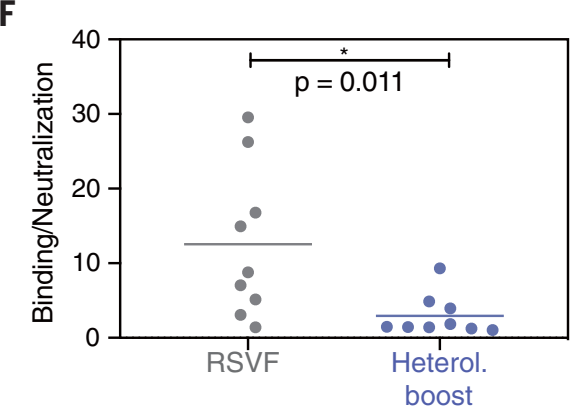

Fig. 5. Immunogenicity of Trivax2 in mice. (A) Three groups of mice ( $n=9$ to 10$)$ were immunized with Trivax2, preRSVF, or heterologous prime boosting as indicated. (B) Binding titers measured against preRSVF using enzyme-linked immunosorbent assay (ELISA). (C) RSV serum-neutralizing titers. (D) Serum composition after three immunizations with Trivax2 or preRSVF. Site-specific responses were determined from serum at day 56 using competition ELISA. (E) Site-specific responses after preRSVF immunization compared with a heterologous prime-boost cohort, as measured by a competition surface plasmon resonance assay. On average, comparing Trivax2 with preRSVF, the response against sites 0, II, and IV increased 4.4-, 2.3-, and 5.3-fold, respectively. (F) Ratio of preRSVF binding to neutralizing antibody titers. Each symbol represents the ratio of binding $\mathrm{IC}_{50}$ to neutralization $\mathrm{IC}_{50}$ for an individual animal; the line represents the mean for each group. Data are representative of at least two independent experiments. Statistics were computed using MannWhitney $U$ test, where ${ }^{*} P<0.05$, ${ }^{* *} P<0.01$, ${ }^{* *} P<0.001$, and ${ }^{*}{ }^{*}{ }^{*} P<0.0001$.
Cocktails of designed immunogens elicit nAbs in vivo and reshape preexisting immunity

Finally, we evaluated the ability of the designed antigens to elicit targeted $\mathrm{nAb}$ responses in vivo. Our rationale for combining site 0, II, and IV immunogens in a cocktail formulation is that all three sites are nonoverlapping in the preRSVF structure (fig. S15) and thus might induce a more potent and consistent nAb response in vivo. To increase immunogenicity, each immunogen was multimerized on selfassembling protein nanoparticles. We chose the RSV nucleoprotein (RSVN), a self-assembling ring-like structure of 10 to 11 subunits that was previously shown to be an effective carrier for the site II immunogen (S2_1.2) (13), and formulated a trivalent immunogen cocktail containing equimolar amounts of S0_1.39, S4 1.5, and S2_1.2 immunogen nanoparticles ("Trivaxl"; fig. S16). The fusion of S0_2.126 and S4_2.45 to RSVN yielded poorly soluble nanoparticles, prompting us to use ferritin particles for multimerization with a $50 \%$ occupancy ( 12 copies), creating a second cocktail comprising S2_1.2 in RSVN and the remaining immunogens in ferritin ("Trivax2"; fig. S17).

In mice, Trivax1 elicited low levels of preRSVF cross-reactive antibodies, and sera did not show RSV-neutralizing activity in most animals (fig. S18). By contrast, immunization with Trivax2 (Fig. 5A) induced robust levels of preRSVF cross-reactive serum levels (Fig. 5B) and six of 10 mice showed neutralizing activity (Fig. 5C). In these mice, the serum antibodybinding responses were equally directed against all three sites (site 0: $32 \pm 6 \%$; site II: $38 \pm 7 \%$; and site IV: $30 \pm 6 \%$ ) (Fig. 5D). This is notable because in previous studies, mice have been a difficult model in which to induce serum neutralization with scaffold-based immunogens $(9,13,28)$. Furthermore, Trivax2 presented only $\sim 14 \%$ of the preRSVF surface area to be targeted by the immune system. Although serum neutralization titers in mice remained substantially lower compared with the titers induced by preRSVF (Fig. 5C), these results demonstrate that vaccine candidates composed of multiple de novo proteins can induce phys- iologically relevant neutralizing serum levels [defined as those with similar or higher in vitro neutralizing activity compared with clinically protective serum concentrations of palivizumab (41)].

Given the well-defined epitope specificities induced by the de novo-designed immunogens, we tested the potential of Trivax 2 to boost sitespecific responses after a priming immunization with preRSVF (Fig. 5A). We found that Trivax2 boosting yielded significantly higher levels of site 0, II, and IV antibodies (4.4-, 2.3-, and 5.3-fold, respectively) compared with boosting immunizations with preRSVF (Fig. 5E). Although overall serum neutralization titers remained inferior to preRSVF boosting (Fig. $5 \mathrm{C})$, Trivax2 boosting resulted in a 4.2 -fold lower ratio of binding to neutralizing antibodies, a common measure to assess vaccineinduced antibody quality $(42,43)$ (Fig. 5F). Therefore, de novo-designed immunogens have the potential to boost site-specific antibodies and to increase the quality of the antibody response compared with repeated boosting immunizations with a viral fusion protein. 
A

$\sum_{\text {SISA }}$ Prime Boost

Day 0 28 $\begin{array}{lll}56 \mathbf{7} & 84\end{array}$

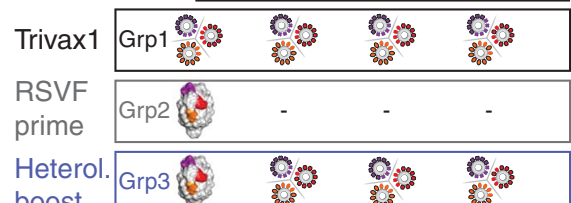

D

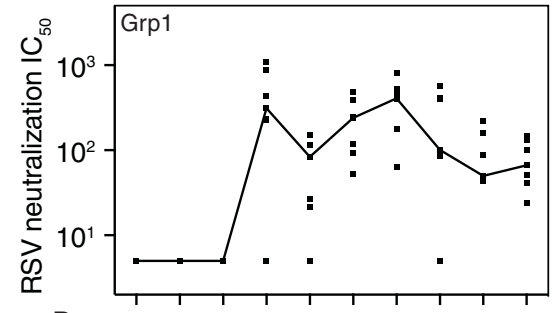

Day $0,1 \times \eta^{8} 3^{0} 5^{0} 6^{3} 8^{1} 9^{1}, 0^{3} \wedge^{19}$
B

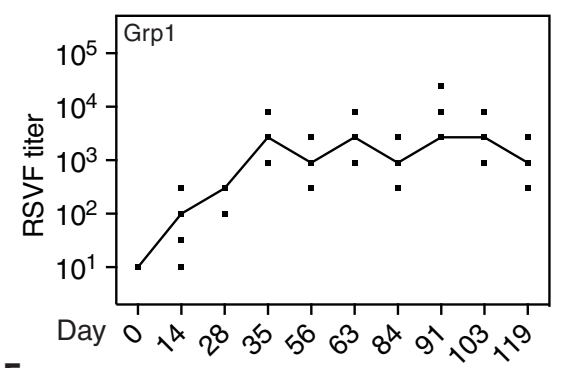

E

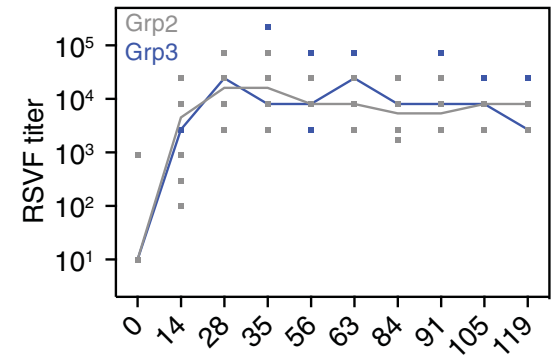

G

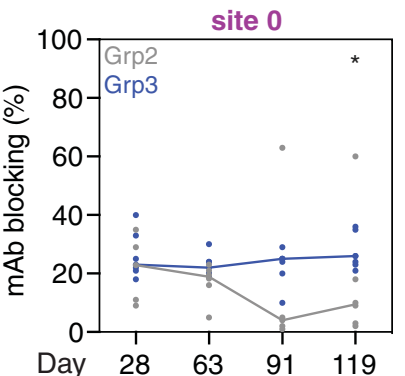

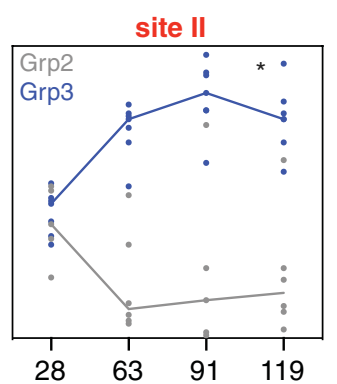

J

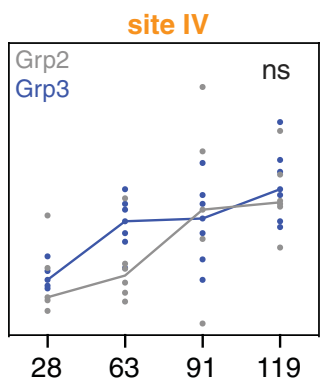

I

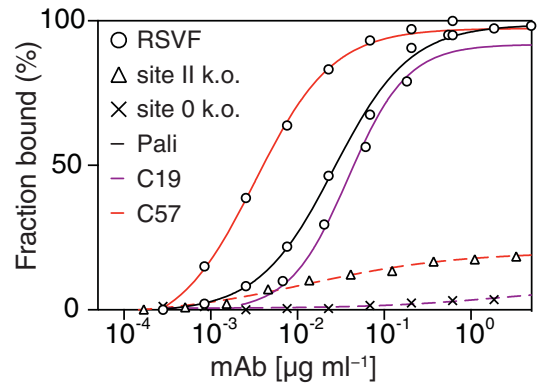

$\mathbf{L}$

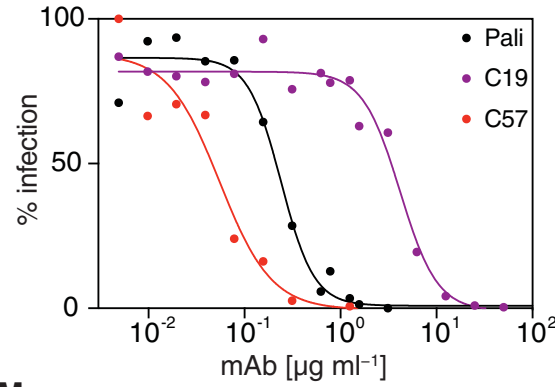

M

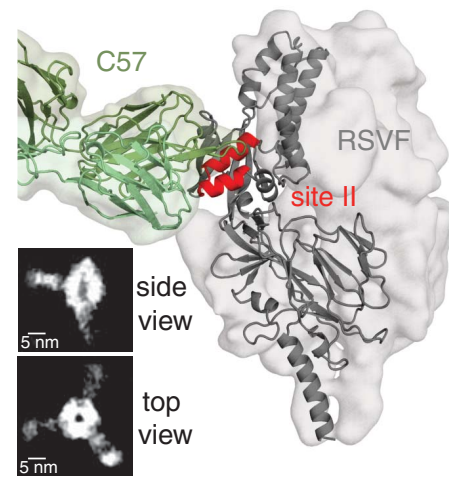

C
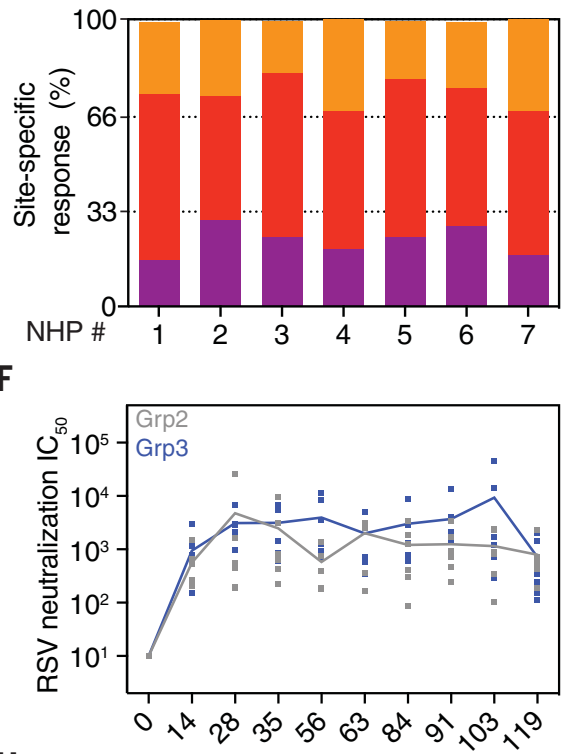

H

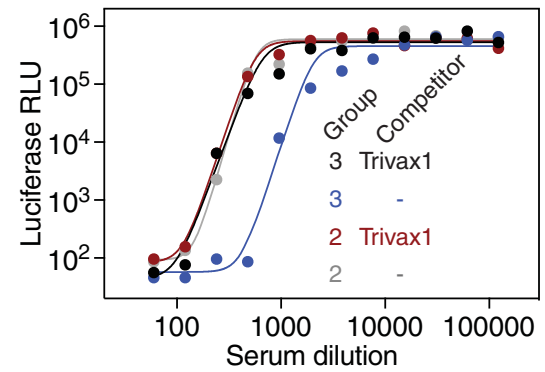

K

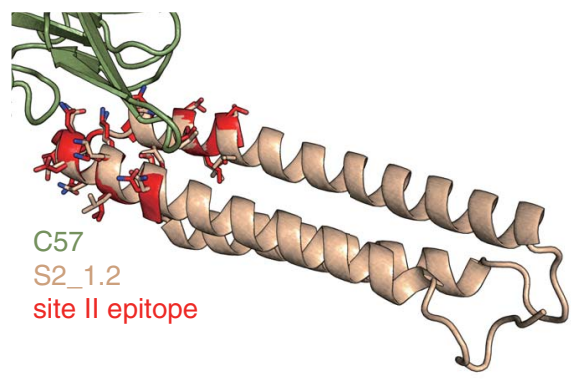

Fig. 6. Immunogenicity of Trivax1 in NHPs. (A) NHP immunization scheme. (B) Trivaxl-immunized NHPs developed robust titers cross-reacting with preRSVF. (C) Sitespecific antibody responses as determined by competition surface plasmon resonance. (D) RSV neutralization titers of group 1. (E) PreRSVF titers and (F) RSV neutralization titers in groups 2 (gray) and 3 (blue). (G) Dynamics of site-specific antibody levels. Site 0 - and site II-specific titers were significantly higher in group 3 compared with group 2 after Trivax1 boosting ( ${ }^{*} P<0.05$, Mann-Whitney $U$ test). (H) RSV neutralization curves upon depletion of day 91 sera with site $0-$, II-, and IV-specific scaffolds.

(I) ELISA binding curves of isolated mAbs $\mathrm{C} 19$ and

C57 to preRSVF and site-specific knockouts compared with palivizumab. (J) In vitro RSV neutralization of C19, C57, and palivizumab. (K) X-ray structure of C57 Fab fragment in complex with S2_1.2. (L) Model of C57 bound to preRSVF, as confirmed by negative-stain electron microscopy. Scale bar, $5 \mathrm{~nm}$. (M) Lineage analysis (Venn diagram) of previously identified site 0 nAbs from three different human donors (39). The elicited site 0 nAb C19 is a close homolog of the human VH5-51 lineage (blue). Data are representative of three independent experiments. 
In parallel, we performed an immunogenicity study in NHPs to test the trivalent cocktail in a closer-to-human antibody repertoire (Fig. 6A). This experiment was designed to test the activity of Trivax1 in both RSV-naïve and preRSVFprimed animals to provide further insights into the ability of computationally designed immunogens to elicit focused antibody responses. The previously designed site II immunogen showed promise in NHPs, but the induced neutralizing titers were low and inconsistent across animals even after repeated immunizations (9). In contrast to mice, all NHPs immunized with Trivax1 developed robust levels of RSVF cross-reactive serum titers (Fig. 6B). Additionally, induced antibodies were directed against all three epitopes (site 0: $23 \pm 5 \%$; site II: $51 \pm 6 \%$; site IV: $25 \pm 5 \%$ ) (Fig. 6C). Six of seven NHPs showed RSVneutralizing serum levels after a single boosting immunization [median inhibitory concentration $\left(\mathrm{IC}_{50}\right)=312$; Fig. $6 \mathrm{D}$ ]. Neutralization titers were maximal at day 84 (median $\mathrm{IC}_{50}$ $=408$ ), and measurements were confirmed by an independent laboratory (fig. S19).

Beyond naïve subjects, an overarching challenge for the development of vaccines against pathogens such as influenza virus, dengue virus, and others is to focus preexisting immunity onto defined neutralization epitopes that can confer long-lasting protection $(13,22,44)$. To mimic a serum response of broad specificity toward RSV, we immunized 13 NHPs with preRSVF. All animals developed strong preRSVFspecific titers (Fig. 6E) and cross-reactivity with all the epitope-focused immunogens, indicating that epitope-specific antibodies were primed against all three epitopes (fig. S20). Six of those preRSVF-primed animals did not receive boosting immunizations so that we could follow the dynamics of epitope-specific antibodies over time (group 2). Seven of the preRSVF-primed animals were boosted three times with Trivax1 (group 3) (Fig. 6A). Although preRSVF-specific antibody and neutralization titers remained statistically comparable in both groups (Fig. 6, $\mathrm{E}$ and F), we found that Trivax1 boosting significantly increased antibodies targeting site II and site 0 , but not site IV (Fig. 6G). In the nonboosted control group, site II and site 0 responses dropped from 37 and $17 \%$ at day 28 to 13 and $4 \%$ at day 91, respectively (Fig. 6G). Compared with the control group, Trivax1 boosting resulted in 6.5-fold higher site II-specific responses on day 91 (84 versus 13\%) and 6.3-fold higher site 0 -specific titers (25 versus $4 \%$ ) (Fig. $6 \mathrm{G})$. By contrast, site IV-specific responses increased to similar levels in both groups, 43 and $40 \%$ in groups 2 and 3, respectively.

To evaluate the functional relevance of reshaping the serum antibody specificities, we depleted site 0-, II-, and IV-specific antibodies from pooled sera. In the Trivaxl-boosted group, we observed a $60 \%$ drop in neutralizing activity compared with only a 7\% drop in the nonboosted control group (Fig. $6 \mathrm{H}$ ). Thus, epitopefocused immunogens can reshape a serum response of broad specificity toward a focused response that predominantly relies on site $0-$, II-, and IV-specific antibodies for RSV neutralization.

Looking further into the molecular basis of the neutralizing activity triggered by the epitope-focused immunogens, we isolated two epitope-specific monoclonal Abs (mAbs) from Trivax1-immunized (group 1) animals using single B cell sorting. Using a panel of binding probes, we confirmed that one mAb targeted antigenic site II (C57) and the other site 0 (C19) (Fig. 6I and fig. S21). Both C57 and $\mathrm{C} 19$ neutralized $\mathrm{RSV}$ in vitro $\left(\mathrm{IC}_{50}=0.03\right.$ and $4.2 \mu \mathrm{g} \mathrm{ml}^{-1}$, respectively). Although C19 was less potent, antibody C57 neutralized RSV with approximately one order of magnitude higher potency compared with the clinically used antibody palivizumab, which is similar to the potency of motavizumab and previously reported antibodies induced by a site II epitope scaffold (9) (Fig. 6J).

To elucidate the molecular basis for the potent site II-mediated neutralization, we solved a crystal structure of C57 in complex with S2_1.2 at a resolution of $2.2 \AA$ (Fig. $6 \mathrm{~K}$ and fig. S13). C57 recognizes the site II epitope in its native conformation, with a full-atom RMSD of $1.21 \AA$ between the epitope in S2_1.2 and site II in preRSVF. Negative-stain electron microscopy of the complex C57-preRSVF further confirmed binding to site II, allowing binding of three Fabs per preRSVF trimer (Fig. 6L).

RSV nAbs are not known for a strictly constrained VH usage as has been described for certain influenza- and HIV-broadly neutralizing antibodies $(45,46)$. However, an important milestone for computationally designed, epitope-focused immunogens is to elicit nAbs similar to those found in humans upon natural infection or vaccination. In one of the largest RSV antibody isolation campaigns, 30 site 0 -specific RSV nAbs were isolated from three human donors (39) derived from 11 different VH genes (Fig. 6M). Of these nAbs, VH5-51 was the only lineage shared among all three donors, suggesting that it is a common precursor for site $0 \mathrm{nAbs}$ in humans. We found that the closest human $\mathrm{VH}$ gene to C19 is indeed the VH5-51 lineage (89\% sequence identity). Thus, computationally designed immunogens can elicit nAbs with properties similar to those found in humans after viral infection (fig. S21).

In summary, the computational design strategies that we have developed enable the design of scaffolds presenting epitopes of unprecedented structural complexity and atomiclevel accuracy. Upon cocktail formulation, the de novo-designed proteins consistently induced RSV neutralization in naïve animals, mediated through three defined epitopes. In addition, the designed antigens were functional in a heterologous prime-boost immunization regimen, inducing more focused antibody responses toward selected, bona fide neutralization epitopes and an overall increase in the quality of the antibody response.

\section{Discussion and conclusions}

Here, we showcased two de novo design strategies for engineering protein scaffolds to present epitopes with high structural complexity. Using template-based de novo design, irregular and discontinuous epitopes were successfully stabilized in heterologous scaffolds. However, this design strategy required extensive optimization by in vitro evolution, and the designs remained suboptimal in their biochemical and biophysical properties. Moreover, this approach lacks the ability to control the topological features of the designed proteins, constituting an important limitation for functional protein design.

To overcome these limitations, we developed TopoBuilder. Compared with other approaches, TopoBuilder has specialized features and advantages to design de novo proteins with structurally complex motifs. First, it assembles topologies tailored to the structural requirements of the functional motif from the start of the design process, rather than through the adaptation (and often destabilization) of a protein structure to accommodate the functional site. Second, the topology assembly results in designed sequences that stably fold and bind with high affinity without requiring iterative rounds of optimization through directed evolution, as is often necessary in computational protein design efforts $(8,47,48)$.

As to the functional aspect of our design work, we have shown that computationally designed immunogens targeting multiple epitopes can induce physiologically relevant levels of functional antibodies in vivo. The elicitation of antibodies targeting conserved epitopes that can mediate broad and potent neutralization remains a central goal for the development of vaccines against pathogens that have frustrated conventional efforts (15). For RSV, the development of a prefusion-stabilized version of RSVF has yielded a superior antigen compared with its postfusion counterpart $(42,49)$, largely attributed to the fact that most preRSVFspecific antibodies are neutralizing $(39,50)$. Given that Trivax only presents a small fraction (14\%) of the antigenic surface of preRSVF, the substantially lower serum titers and consequently lower bulk serum neutralization elicited by Trivax may be expected and will likely require substantial optimization in terms of delivery and formulation to increase the magnitude of the response.

Nevertheless, we have shown that a cocktail of de novo-designed proteins induced relevant levels of serum neutralization in most naïve 
mice and NHPs. Beyond bulk serum titers, Trivax offers an unprecedented level of control over antibody specificities to the single-epitope level. In heterologous prime-boost immunization schemes, Trivax profoundly reshaped the serum composition, leading to increased levels of desirable antibody specificities and an overall improved quality of the antibody response. The ability to selectively boost subdominant nAbs targeting defined, broadly protective epitopes could overcome long-standing obstacles in the development of vaccines against pathogens such as influenza, for which the challenge is to overcome established immunodominance hierarchies (51).

This study provides a blueprint for the computational design of epitope-focused vaccines. In addition to antigens for viral epitopes, the ability to stabilize structurally complex epitopes in a de novo protein with a defined protein topology may prove useful in eliciting and isolating mAbs against tertiary epitopes with potentially exclusive allosteric or therapeutic properties. Beyond immunogens, our work presents an alternate approach for the design of de novo functional proteins, enabling the assembly of customized protein topologies tailored to structural and functional requirements of the motif. The ability to design de novo proteins presenting functional sites with high structural complexity will be broadly applicable to expanding the structural and sequence repertoires and, above all, the functional landscape of natural proteins.

\section{REFERENCES AND NOTES}

1. N. Koga et al., Principles for designing ideal protein structures. Nature 491, 222-227 (2012). doi: 10.1038/nature11600; pmid: 23135467

2. E. Marcos et al., Principles for designing proteins with cavities formed by curved $\beta$ sheets. Science 355, 201-206 (2017). doi: 10.1126/science.aah7389; pmid: 28082595

3. P. S. Huang et al., De novo design of a four-fold symmetric TIM-barrel protein with atomic-level accuracy. Nat. Chem. Biol. 12, 29-34 (2016). doi: 10.1038/nchembio.1966; pmid: 26595462

4. M. Mravic et al., Packing of apolar side chains enables accurate design of highly stable membrane proteins. Science 363 1418-1423 (2019). doi: 10.1126/science.aav7541; pmid: 30923216

5. P. S. Huang, S. E. Boyken, D. Baker, The coming of age of de novo protein design. Nature 537, 320-327 (2016). doi: 10.1038/nature19946; pmid: 27629638

6. W. M. Dawson, G. G. Rhys, D. N. Woolfson, Towards functional de novo designed proteins. Curr. Opin. Chem. Biol. 52, 102-111 (2019). doi: 10.1016/j.cbpa.2019.06.011; pmid: 31336332

7. A. Chevalier et al., Massively parallel de novo protein design for targeted therapeutics. Nature 550, 74-79 (2017). doi: 10.1038/nature23912; pmid: 28953867

8. E. Procko et al., A computationally designed inhibitor of an Epstein-Barr viral Bcl-2 protein induces apoptosis in infected cells. Cell 157, 1644-1656 (2014). doi: 10.1016/ j.cell.2014.04.034; pmid: 24949974

9. B. E. Correia et al., Proof of principle for epitope-focused vaccine design. Nature 507, 201-206 (2014). doi: 10.1038/ nature12966; pmid: 24499818

10. S. Jones, J. M. Thornton, Principles of protein-protein interactions. Proc. Natl. Acad. Sci. U.S.A. 93, 13-20 (1996) doi: 10.1073/pnas.93.1.13; pmid: 8552589

11. N. D. Rubinstein et al., Computational characterization of B-cell epitopes. Mol. Immunol. 45, 3477-3489 (2008) doi: 10.1016/j.molimm.2007.10.016; pmid: 18023478
12. G. L. Holliday, J. D. Fischer, J. B. Mitchell, J. M. Thornton, Characterizing the complexity of enzymes on the basis of their mechanisms and structures with a bio-computational analysis. FEBS J. 278, 3835-3845 (2011). doi: 10.1111/j.17424658.2011.08190.x; pmid: 21605342

13. F. Sesterhenn et al., Boosting subdominant neutralizing antibody responses with a computationally designed epitopefocused immunogen. PLOS Biol. 17, e3000164 (2019) doi: 10.1371/journal.pbio.3000164; pmid: 30789898

14. D. R. Burton, What are the most powerful immunogen design vaccine strategies? Reverse vaccinology 2.0 shows great promise. Cold Spring Harb. Perspect. Biol. 9, a030262 (2017). doi: 10.1101/cshperspect.a030262; pmid: 28159875

15. R. Rappuoli, M. J. Bottomley, U. D'Oro, O. Finco, E. De Gregorio , Reverse vaccinology 2.0: Human immunology instructs vaccine antigen design. J. Exp. Med. 213, 469-481 (2016). doi: 10.1084/jem.20151960; pmid: 27022144

16. F. Sesterhenn, J. Bonet, B. E. Correia, Structure-based immunogen design-leading the way to the new age of precision vaccines. Curr. Opin. Struct. Biol. 51, 163-169 (2018) doi: 10.1016/j.sbi.2018.06.002; pmid: 29980105

17. K. E. Neu, C. J. Henry Dunand, P. C. Wilson, Heads, stalks and everything else: How can antibodies eradicate influenza as a human disease? Curr. Opin. Immunol. 42, 48-55 (2016). doi: 10.1016/j.coi.2016.05.012; pmid: 27268395

18. D. Sok, D. R. Burton, Recent progress in broadly neutralizing antibodies to HIV. Nat. Immunol. 19, 1179-1188 (2018). doi: 10.1038/s41590-018-0235-7; pmid: 30333615

19. W. Dejnirattisai et al., Dengue virus sero-cross-reactivity drives antibody-dependent enhancement of infection with zika virus. Nat. Immunol. 17, 1102-1108 (2016). doi: 10.1038/ni.3515; pmid: 27339099

20. J. G. Jardine et al., HIV-1 broadly neutralizing antibody precursor B cells revealed by germline-targeting immunogen. Science 351, 1458-1463 (2016). doi: 10.1126/science.aad9195; pmid: 27013733

21. Y. Avnir et al., Molecular signatures of hemagglutinin stemdirected heterosubtypic human neutralizing antibodies against influenza A viruses. PLOS Pathog. 10, e1004103 (2014). doi: 10.1371/journal.ppat.1004103; pmid: 24788925

22. G. Barba-Spaeth et al., Structural basis of potent Zika-dengue virus antibody cross-neutralization. Nature 536, 48-53 (2016). doi: 10.1038/nature18938; pmid: 27338953

23. D. Corti et al., Cross-neutralization of four paramyxoviruses by a human monoclonal antibody. Nature 501, 439-443 (2013). doi: 10.1038/nature12442; pmid: 23955151

24. D. Corti et al., A neutralizing antibody selected from plasma cells that binds to group 1 and group 2 influenza A hemagglutinins. Science 333, 850-856 (2011). doi: 10.1126/ science.1205669; pmid: 21798894

25. K. Xu et al., Epitope-based vaccine design yields fusion peptide-directed antibodies that neutralize diverse strains of HIV-1. Nat. Med. 24, 857-867 (2018). doi: 10.1038/s41591018-0042-6: pmid: 29867235

26. G. Ofek et al., Elicitation of structure-specific antibodies by epitope scaffolds. Proc. Natl. Acad. Sci. U.S.A. 107 17880-17887 (2010). doi: 10.1073/pnas.1004728107; pmid: 20876137

27. B. E. Correia et al., Computational design of epitope-scaffolds allows induction of antibodies specific for a poorly immunogenic HIV vaccine epitope. Structure 18, 1116-1126 (2010). doi: 10.1016/j.str.2010.06.010; pmid: 20826338

28. J. S. McLellan et al., Design and characterization of epitope-scaffold immunogens that present the motavizumab epitope from respiratory syncytial virus. J. Mol. Biol. 409, 853-866 (2011). doi: 10.1016/j.jmb.2011.04.044: pmid: 21549714

29. J. S. McLellan et al., Structure of RSV fusion glycoprotein trimer bound to a prefusion-specific neutralizing antibody. Science 340, 1113-1117 (2013). doi: 10.1126/science.1234914; pmid: 23618766

30. J. S. McLellan et al., Structure of a major antigenic site on the respiratory syncytial virus fusion glycoprotein in complex with neutralizing antibody 101F. J. Virol. 84, 12236-12244 (2010). doi: 10.1128/JVI.01579-10; pmid: 20881049

31. M. L. Azoitei et al., Computation-guided backbone grafting of a discontinuous motif onto a protein scaffold. Science 334. 373-376 (2011). doi: 10.1126/science.1209368: pmid: 22021856

32. S. J. Fleishman et al., Computational design of proteins targeting the conserved stem region of influenza hemagglutinin. Science 332, 816-821 (2011). doi: 10.1126/ science.1202617; pmid: 21566186
33. D. Tian et al., Structural basis of respiratory syncytial virus subtype-dependent neutralization by an antibody targeting the fusion glycoprotein. Nat. Commun. 8, 1877 (2017). doi: 10.1038/s41467-017-01858-w; pmid: 29187732

34. J. Bonet et al., Rosetta FunFolDes - A general framework for the computational design of functional proteins. PLOS Comput. Biol. 14, e1006623 (2018). doi: 10.1371/journal.pcbi.1006623; pmid: 30452434

35. T. J. Brunette et al., Exploring the repeat protein universe through computational protein design. Nature 528, 580-584 (2015). doi: 10.1038/nature16162; pmid: 26675729

36. W. R. Taylor, A 'periodic table' for protein structures. Nature 416, 657-660 (2002). doi: 10.1038/416657a; pmid: 11948354

37. D. N. Woolfson et al., De novo protein design: How do we expand into the universe of possible protein structures? Curr. Opin. Struct. Biol. 33, 16-26 (2015). doi: 10.1016/ j.sbi.2015.05.009; pmid: 26093060

38. M. D. Finucane, M. Tuna, J. H. Lees, D. N. Woolfson, Coredirected protein design. I. An experimental method for selecting stable proteins from combinatorial libraries. Biochemistry 38, 11604-11612 (1999). doi: 10.1021/bi990765n; pmid: 10512615

39. M. S. Gilman et al., Rapid profiling of RSV antibody repertoires from the memory $B$ cells of naturally infected adult donors. Sci. Immunol. 1, eaaj1879 (2016). doi: 10.1126/sciimmunol. aaj1879; pmid: 28111638

40. A. M. Watkins, P. S. Arora, Anatomy of $\beta$-strands at proteinprotein interfaces. ACS Chem. Biol. 9, 1747-1754 (2014). doi: 10.1021/cb500241y; pmid: 24870802

41. C. Fenton, L. J. Scott, G. L. Plosker, Palivizumab: A review of its use as prophylaxis for serious respiratory syncytial virus infection. Paediatr. Drugs 6, 177-197 (2004). doi: 10.2165/ 00148581-200406030-00004; pmid: 15170364

42. M. C. Crank et al., VRC 317 Study Team, A proof of concept for structure-based vaccine design targeting RSV in humans. Science 365, 505-509 (2019). doi: 10.1126/science.aav9033; pmid: 31371616

43. J. Marcandalli et al., Induction of potent neutralizing antibody responses by a designed protein nanoparticle vaccine for respiratory syncytial virus. Cell 176, 1420-1431.e17 (2019). doi: 10.1016/j.cell.2019.01.046; pmid: 30849373

44. S. F. Andrews et al., Immune history profoundly affects broadly protective B cell responses to influenza. Sci. Transl. Med. 7, 316ra192 (2015). doi: 10.1126/scitranslmed.aad0522; pmid: 26631631

45. D. Lingwood et al., Structural and genetic basis for development of broadly neutralizing influenza antibodies. Nature 489, 566-570 (2012). doi: 10.1038/naturel1371; pmid: 22932267

46. A. P. West Jr., R. Diskin, M. C. Nussenzweig, P. J. Bjorkman, Structural basis for germ-line gene usage of a potent class of antibodies targeting the CD4-binding site of HIV-1 gp120. Proc. Natl. Acad. Sci. U.S.A. 109, E2083-E2090 (2012). doi: 10.1073/pnas.1208984109; pmid: 22745174

47. D. A. Silva et al., De novo design of potent and selective mimics of IL-2 and IL-15. Nature 565, 186-191 (2019). doi: 10.1038/ s41586-018-0830-7; pmid: 30626941

48. E. M. Strauch et al., Computational design of trimeric influenzaneutralizing proteins targeting the hemagglutinin receptor binding site. Nat. Biotechnol. 35, 667-671 (2017). doi: 10.1038/ nbt.3907; pmid: 28604661

49. J. S. McLellan et al., Structure-based design of a fusion glycoprotein vaccine for respiratory syncytial virus. Science 342, 592-598 (2013). doi: 10.1126/science.1243283; pmid: 24179220

50. J. 0. Ngwuta et al.. Prefusion F-specific antibodies determine the magnitude of RSV neutralizing activity in human sera. Sci. Transl. Med. 7, 309ra162 (2015). doi: 10.1126/ scitransImed.aac4241; pmid: 26468324

51. D. Angeletti et al., Defining B cell immunodominance to viruses. Nat. Immunol. 18, 456-463 (2017). doi: 10.1038/ ni.3680; pmid: 28192417

52. F. Sesterhenn, C. Yang, J. Bonet, B. E. Correia, LPDI-EPFL/ trivalent_cocktail: RSV_trivalent_cocktail, Zenodo (2020); https://doi.org/10.5281/zenodo.3724331.

\section{ACKNOWLEDGMENTS}

We thank W. R. Schief, P. Gainza, S. T. Reddy, and B. Lemaitre for helpful discussions and comments on the manuscript; J. E. Crowe, Jr., for providing site IV antibodies; A. McCarthy at ESRF for beam line support; the Behavioral Science Foundation (H. Hotchin, A. Beierschmitt, and R. Palmour) for the NHP immunization and PBMC isolation; and ExcellGene (Monthey, Switzerland) for help 
with mammalian protein expression. We also thank several EPFL facilities: PTPSP (K. Lau, A. Reynaud, F. Pojer, D. Hacker, L. Durrer and S. Ouinche) for protein expression and crystallography support; the phenogenomics center (C. Waldvogel, R. Doenlen) for support with mouse experiments; CIME and PTBIOEM (D. Demurtas and S. Nazarov) for electron microscopy support; the flow cytometry core facility; the gene expression core facility for support with next-generation sequencing; and SCITAS for support in high performance computing. The computational simulations were also partially facilitated by the CSCS Swiss National Supercomputing Centre. Funding: This work was supported by the Swiss Initiative for Systems Biology (SystemsX.ch), the European Research Council (Starting Grant 716058), the Swiss National Science Foundation (310030_163139), and the EPFL's Catalyze4Life initiative. F.S. was supported by an SNF/Innosuisse BRIDGE Proof-of-Concept Grant. J.B. was supported by an EPFL Fellows Postdoctoral Fellowship. T.K. received funding from the German Center of Infection Research (DZIF) and the Cluster of Excellence RESIST (EXC 2155) of the German Research Foundation. J.T.C. was funded by ERA-Net Prionlmmunity project $01 G M 1503$ (Federal Ministry of Education and Research, Germany). V.M. received funding from AESI-18 (Instituto de Salud Carlos III grant MPY 375/18). J.-P.J. received funding from the Canada Research Chairs program. T.J. and X.W. received funding from
NIH NIAID R01 Al137523. The funders had no role in study design, data collection and analysis, decision to publish, or preparation of the manuscript. Author contributions: F.S., C.Y., and B.E.C. conceived the work and designed the experiments. F.S. and C.Y. performed the computational design and the experimental characterizations. J.B. developed the TopoBuilder. J.T.C., C.Y.,

G.C., T.K., X.W., and T.J. solved the $x$-ray structures. L.A.A. performed NMR experiments and solved the NMR structure. Y.W., C.-I.C., and Y.L. isolated mAbs from NHPS. I.K. and J.-P.J. performed and analyzed samples by electron microscopy. S.S.V., M.G., S.R., P.C., S.G., M.V., C.-A.R., E.D., E.O., D.D., T.D., V.M., J.-F.E., and M.A.R.-W. performed experiments and analyzed data. J.T.B., S.E., and S.R. contributed to the design and planning of animal studies. F.S., C.Y., and B.E.C. wrote the manuscript with input from all authors. Competing interests: B.E.C., F.S.,

C.Y., and J.B. are inventors on a patent application by the École Polytechnique fédérale de Lausanne that covers the designed antigens. S.R. and J.-F.E. are inventors on two patents filed by the Institut National de la Recherche Agronomique (INRA) that covers the use of the $\mathrm{N}$ protein as antigen carrier for vaccines. Data and materials availability: All code used for this study is available through a public GitHub repository: https://github.com/ Ipdi-epfl/trivalent_cocktail and are archived in Zenodo (52). Structures have been deposited in the Protein Data Bank under accession codes 6S3D (S0 2126 in complex with D25), 6XWI (S0_2.126 solution NMR structure), 6XXV (S2_1.2 in complex with the Fab fragment of the elicited antibody C57), and 6VTW (S4 2.45 in complex with 101F). Chemical shift data for SO_2.126 were deposited in the Biological Magnetic Resonance Data Bank under accession code 34481. The plasmids of the designed proteins are available from the authors under a material transfer agreement with the Ecole Polytechnique Fédérale de Lausanne (EPFL). All other data needed to evaluate the conclusions in this paper are present either in the main text or the supplementary materials.

\section{SUPPLEMENTARY MATERIALS}

science.sciencemag.org/content/368/6492/eaay5051/suppl/DC1 Materials and Methods

Tables S1 to S8

Figs. S1 to S21

References (53-83)

View/request a protocol for this paper from Bio-protocol.

24 June 2019; resubmitted 30 January 2020

Accepted 8 April 2020

10.1126/science.aay5051 


\section{Science}

\section{De novo protein design enables the precise induction of RSV-neutralizing antibodies}

Fabian Sesterhenn, Che Yang, Jaume Bonet, Johannes T. Cramer, Xiaolin Wen, Yimeng Wang, Chi-I Chiang, Luciano A. Abriata, Iga Kucharska, Giacomo Castoro, Sabrina S. Vollers, Marie Galloux, Elie Dheilly, Stéphane Rosset, Patricia Corthésy, Sandrine Georgeon, Mélanie Villard, Charles-Adrien Richard, Delphyne Descamps, Teresa Delgado, Elisa Oricchio, Marie-Anne Rameix-Welti, Vicente Más, Sean Ervin, Jean-François Eléouët, Sabine Riffault, John T. Bates, Jean-Philippe Julien, Yuxing Li, Theodore Jardetzky, Thomas Krey and Bruno E. Correia

Science 368 (6492), eaay5051.

DOI: $10.1126 /$ science.aay5051

\section{TopoBuilding precision vaccines}

To induce strong and targeted neutralizing antibody $(n A b)$ responses against vaccine targets, one strategy has been to use computationally designed immunogens. However, the structural complexity of many known neutralization epitopes has posed a major challenge for the design of accurate epitope mimetics. Sesterhenn et al. created a protein design algorithm called TopoBuilder to design scaffolds for irregular and discontinuous neutralization epitopes. As a proof of principle, the authors generated epitope-focused immunogens based on the prefusion conformation of the respiratory syncytial virus (RSV) fusion protein. When these immunogens were used to vaccinate mice and nonhuman primates in RSV infection models, they generated targeted nAb responses to RSV and boosted site-specific nAb responses in heterologous prime-boost vaccination schemes. Science, this issue p. eaay 5051

ARTICLE TOOLS

SUPPLEMENTARY MATERIALS

RELATED

REFERENCES

PERMISSIONS http://science.sciencemag.org/content/368/6492/eaay5051

http://science.sciencemag.org/content/suppl/2020/05/13/368.6492.eaay5051.DC1

http://stm.sciencemag.org/content/scitransmed/12/540/eaaw0268.full

http://stm.sciencemag.org/content/scitransmed/9/388/eaaj1928.full

http://stm.sciencemag.org/content/scitransmed/7/312/312ra175.full

http://stm.sciencemag.org/content/scitransmed/7/309/309ra162.full

This article cites 82 articles, 23 of which you can access for free http://science.sciencemag.org/content/368/6492/eaay5051\#BIBL

http://www.sciencemag.org/help/reprints-and-permissions

Use of this article is subject to the Terms of Service

Science (print ISSN 0036-8075; online ISSN 1095-9203) is published by the American Association for the Advancement of Science, 1200 New York Avenue NW, Washington, DC 20005. The title Science is a registered trademark of AAAS.

Copyright (C) 2020 The Authors, some rights reserved; exclusive licensee American Association for the Advancement of Science. No claim to original U.S. Government Works 